\title{
Egg intake and cancers of the breast, ovary and prostate: a dose-response meta-analysis of prospective observational studies
}

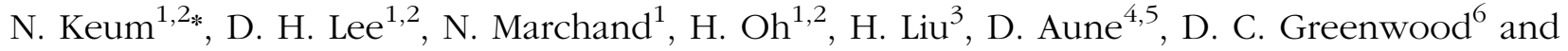 \\ E. L. Giovannucci ${ }^{1,2,7}$ \\ ${ }^{1}$ Department of Nutrition, Harvard T.H. Chan School of Public Health, Boston, USA \\ ${ }^{2}$ Department of Epidemiology, Harvard T.H. Chan School of Public Health, Boston, USA \\ ${ }^{3}$ Department of Epidemiology and Health Statistics, Zhejiang University, Zhejiang, People's Republic of China \\ ${ }^{4}$ Department of Public Health and General Practice, Faculty of Medicine, Norwegian University of Science and Technology, \\ Trondheim, Norway \\ ${ }^{5}$ Department of Epidemiology and Biostatistics, Imperial College London, London, UK \\ ${ }^{6}$ Division of Biostatistics, University of Leeds, Leeds, UK \\ ${ }^{7}$ Channing Division of Network Medicine, Department of Medicine, Brigham and Women's Hospital and Harvard Medical \\ School, Boston, MA, USA \\ (Submitted 27 January 2015 - Final revision received 1 May 2015 - Accepted 20 May 2015 - First published online 21 August 2015)
}

\section{Abstract}

Evidence suggests that egg intake may be implicated in the aetiology of sex hormone-related cancers. However, dose-response relationships between egg intake and such cancers are unclear. Thus, we conducted a dose-response meta-analysis to summarise the dose-response relationships between egg consumption and the risk of breast, prostate and gynaecological cancers. A literature search was performed using PubMed and Embase up to April 2015 to identify relevant prospective observational studies. Summary relative risk (RR) and 95\% CI were estimated using a random-effects model. For breast cancer, the linear dose-response meta-analysis found a non-significantly increased risk (RR for an increase of 5 eggs consumed/week: 1.05, 95\% CI 0.99, 1.11, $n$ 16023 cases). Evidence for non-linearity was not statistically significant $\left(P_{\text {non-linearity }}=0 \cdot 50, n 15415\right.$ cases) but consuming $\geq 5$ eggs/week was significantly associated with an increased risk of breast cancer compared with no egg consumption, with the summary RR being 1.04 (95\% CI 1.01, 1.07) for consuming 5 eggs/week and 1.09 (95\% CI 1.03, 1.15) for consuming about 9 eggs/week. For other cancers investigated, the summary RR for an increase of 5 eggs consumed/week was 1.09 (95\% CI 0.96, 1.24, $n 2636$ cases) for ovarian cancer; 1.47 (95\% CI 1.01, 2.14, $n 609$ cases) for fatal prostate cancer, with evidence of small-study effects $\left(P_{\text {Egger }}=0.04\right)$. No evidence was found for an association with the risk of total prostate cancer. While our conclusion was tempered by the potential for publication bias and confounding, high egg intake may be associated with a modestly elevated risk of breast cancer, and a positive association between egg intake and ovarian and fatal prostate cancers cannot be ruled out.

\section{Key words: Egg intake: Breast cancer: Ovarian cancer: Prostate cancer: Dose-response meta-analysis: Cohort studies}

Eggs are frequently consumed worldwide, with an estimated number of eggs consumed per person over a year ranging from a low of forty-seven in India to a high of 349 in China in 2007 (interestingly, 250 in the USA and 178 in the UK) ${ }^{(1)}$. Eggs are low in saturated fat and have a high nutritional value supplying high-quality protein, mono- and polyunsaturated fats, vitamins (A, B and D) and minerals (e.g. Fe) ${ }^{(2)}$. However, their high contents of cholesterol $\left(186 \mathrm{mg}\right.$ per $50 \mathrm{~g} \mathrm{egg}^{(2)}$ v. $300 \mathrm{mg}$ reference daily intake ${ }^{(3)}$ ) and choline (126 mg per $50 \mathrm{~g} \mathrm{egg}^{(2)}$ v. 425-550 mg/d of adequate intake for women and men aged $\geq 19$ years ${ }^{(4)}$ ) lend biological plausibility to a possible role of egg consumption in the aetiology of sex hormone-related cancers, especially for those whose circulating levels of cholesterol and choline are sensitive to dietary intake of the nutrients. Cholesterol serves as a precursor for the biosynthesis of sex hormones such as androgens and oestrogens $^{(5)}$ that promote cell proliferation, thereby contributing to carcinogenesis of breast, prostate and gynaecological cancers $^{(6-8)}$. Evidence also suggests that 27-hydroxycholesterol, a primary metabolite of cholesterol, acts as an oestrogen

Abbreviations: AICR, American Institute for Cancer Research; RR, relative risk; WRCF, World Cancer Research Fund.

*Corresponding author: N. Keum, fax +1617432 2435, email nak212@mail.harvard.edu 
receptor agonist in breast cancer cells, mimicking the effects of oestrogen ${ }^{(9,10)}$. Choline has been particularly implicated in the proliferation and progression of prostate cancer through its role in cell membrane synthesis and function ${ }^{(11)}$, although choline, as a methyl donor, has been suggested to be inversely associated with the risk of breast cancer ${ }^{(12)}$. Furthermore, given that a $50 \mathrm{~g}$ egg contains only 78 calories $^{(2)}$, eggs provide a considerable amount of protein per energy content $(6 \mathrm{~g}$ per 78 calories $^{(2)} v .50 \mathrm{~g}$ reference daily intake $\left.{ }^{(3)}\right)$. High protein intake, particularly high-quality proteins ${ }^{(13,14)}$, increases the production of insulin-like growth factor 1 that promotes tissue growth and tumour progression ${ }^{(13,15,16)}$. Lastly, chlorine that is used to wash eggs before commercial sale may enter eggs through the pores in the shells and interact with eggs' organic substances, converting to potentially carcinogenic organochlorines. Organochlorines disrupt oestrogen-related pathways, thereby implicated in the pathogenesis of gynaecological cancers and, particularly, breast cancer ${ }^{(17)}$.

Indeed, several meta-analyses of observational studies have assessed the potential harm of egg intake on such cancers and found a positive association with the risk of breast ${ }^{(18)}$ and ovarian $^{(19)}$ cancers, suggested some association with an increased risk of endometrial ${ }^{(20)}$ and fatal prostate ${ }^{(21)}$ cancers, but found no association with the risk of total prostate cancer $^{(21)}$. However, given that the distribution of egg intake differs across studies, previous meta-analyses ${ }^{(19-21)}$ that pooled RR comparing the highest with the lowest category of egg intake have limited interpretability in terms of the dose-response relationship.

To better guide dietary recommendations for egg intake, it remains to be answered whether the risk of cancer increases with each additional egg intake, or whether there is a range of egg intake that does not elevate the risk of cancer. While the meta-analysis on breast cancer ${ }^{(18)}$ has addressed the aforementioned problem by pooling RR separately for a distinctive range of egg intake (e.g. $1-<2 v .<1,2-5 v .<1$, $>5 v .<1 \mathrm{egg} /$ week), it included case-control studies with a high potential for recall and selection bias to distort the true diet-cancer relationship. The World Cancer Research Fund/American Institute for Cancer Research (WRCF/AICR) Expert Panel assessed the dose-response relationships between egg intake and cancers of the ovary ${ }^{(22)}$ and prostate $^{(23)}$ using prospective studies published up to December 2012 and April 2013, respectively, but the evidence for an association was evaluated as 'limited - no conclusion'. More studies may have been published since then and updating analyses incorporating them will help better evaluate the evidence. Therefore, based on prospective observational studies, we conducted a dose-response meta-analysis to quantify the amount of egg intake associated with an increase in the risk of breast, prostate and gynaecological cancers, and to identify the shape of doseresponse relationships.

\section{Methods}

For the design, analysis and reporting of the present study, standard guidelines for the meta-analysis of prospective observational studies were followed ${ }^{(24)}$. Literature search, study selection and data extraction were performed independently by three authors (D. H. L., H. L. and N. M.). Inconsistency between the researchers was resolved through discussion.

\section{Literature search}

PubMed and Embase databases were searched for studies published up to April 2015. Detailed search terms are provided in online Supplementary Table S1. The language was limited to English and no other restrictions were imposed. Abstracts and unpublished results were not included. The reference lists of selected reviews and meta-analyses, and all the articles included in the present study were also reviewed for additional studies.

\section{Study selection}

Studies were selected if they were a prospective observational study (i.e. a cohort study analysed with nested case-control, case-cohort or prospective cohort approaches) or a pooled study analysed prospectively on the relationship between egg intake and incidence of breast, prostate and gynaecological cancers. A pooled study was considered for inclusion because not all cohort studies included in a pooled study published the results independently. Retrospective case-control studies were excluded to minimise the impact of recall bias and selection bias. Of note, prostate cancer is a highly heterogeneous disease and overdetection of indolent prostate cancer often dilutes the exposure and outcome relationship in epidemiological studies. Therefore, we also included studies reporting on fatal prostate cancer in order to examine the effect of egg intake on aggressive prostate cancer separately.

For the dose-response meta-analysis, studies were selected if they provided the following information: a quantitative measure (e.g. g/d or week, numbers/d or week, servings/d or week) of egg intake for at least three categories with the estimates of relative risks (RR) (rate ratio or hazard ratio), 95\% CI, category-specific or total number of cases, and category-specific or total number of either non-cases or person-years. When studies did not provide the aforementioned information but directly reported RR and 95\% CI for the linear effect of egg intake, they were included in the linear dose-response meta-analysis. Authors of two studies $^{(25,26)}$ were contacted to obtain data on categoryspecific number of person-years or cases, and they provided the requested information.

\section{Data extraction}

From each study, the following information was extracted: the most fully adjusted RR and corresponding 95\% CI in each category of egg intake; category-specific range of egg intake; unit of egg intake; category-specific or total number of cases; category-specific or total number of person-years or non-cases; first author's name; publication year; study design; study period; characteristics of the study population 
(cohort name, country, sex and age at enrolment); dietary assessment method (type and whether validated or not); adjustment variables.

\section{Statistical analysis}

The dose-response meta-analysis consists of two parts: linear analysis and non-linear analysis. For the linear dose-response meta-analysis, assuming a linear relationship between egg intake and the risk of cancers, the method described by Greenland \& Longnecker ${ }^{(27)}$ was used to calculate studyspecific RR (linear slopes) and 95\% CI from the correlated RR and 95\% CI extracted across the categories of egg intake. To estimate study-specific linear trends, several approximations were made: the midpoint of egg intake in each category was assigned to the corresponding RR; the width of the open-ended highest category was assumed to be the same as that of the adjacent interval; when the distributions of person-years or non-cases were not provided but analysed based on quantiles, they were equally divided across the quantiles. Egg intake reported in servings or $\mathrm{g} / \mathrm{d}$ or week was converted to number of eggs/week, by assuming the weight of one egg as $50 \mathrm{~g}$ (equivalent to one large egg) and the portion size of one serving as one egg. Then, the estimated study-specific RR and variance were pooled using the DerSimonian-Laird random-effects model to calculate the summary RR and 95\% CI. Forest plots of the linear doseresponse meta-analysis are presented for RR for an increase of 5 eggs consumed/week.

To explore the potential non-linear relationship between egg intake and the risk of cancers, the non-linear doseresponse meta-analysis was performed based on the restricted cubic spline approach ${ }^{(28,29)}$. For each study, cubic splines were modelled with three knots fixed at percentiles (10, 50 and $90 \%$ ) of the whole distribution of egg intake contributed by all the included studies, accounting for correlation across the category-specific RR and 95\% CI within each study ${ }^{(28)}$. The reference was set to 0 eggs/d, the lowest value of the reported egg intake. Then, the derived curves were combined using the multivariate DerSimonian-Laird random-effects meta-analysis ${ }^{(30)}$. The $P$ value for non-linearity was obtained from the test of the null hypothesis that the regression coefficient of the second spline transformation was equal to zero. Of note, the cubic spline approach requires that studies analyse egg intake in more than three categories. To allow adequate information for the robust estimation of the curve, this non-linear meta-analysis was applied when five or more cohort studies contributed data to the non-linear doseresponse meta-analysis.

Heterogeneity in the relationship between egg intake and the risk of cancers across the studies was assessed by Cochran's $Q$ test $^{(31)}$, and quantified by the percentage of total variation across the studies that is attributable to between-study heterogeneity $\left(I^{2}\right)^{(32)}$. No subgroup analyses and meta-regression were conducted due to the small number of studies included and upon observing no evidence of heterogeneity in most of the analyses. The potential for small-study effects $^{(33,34)}$, such as publication bias, was assessed visually using the funnel plot and statistically using Egger's test ${ }^{(35)}$, knowing that the statistical power is limited when the number of studies included is small. To explore the robustness of the results, diverse sensitivity analyses were performed such as assuming one serving size of egg intake as two eggs for studies that did not report or did not specify the serving size of egg intake on FFQ and assessing the influence of individual studies on the pooled estimate by excluding each study in turn. For statistical significance, twosided $\alpha$ was set at $0 \cdot 05$. All statistical analyses were conducted using STATA 12 (StataCorp).

\section{Results}

The results of the literature search and study selection are summarised in Fig. 1. Of the 7378 publications screened, a total of eighteen $^{(25,26,36-51)}$ were included in the present doseresponse meta-analysis. For gynaecological cancers, no studies met the inclusion criteria, except for ovarian cancer. Thus, the present dose-response meta-analysis examined three cancer sites (breast, ovary and prostate) in relation to egg intake.

The characteristics of the eighteen studies are summarised in online Supplementary Table S2. Of the eighteen publications, six were on breast cancer, of which one was a pooled study. Among the breast cancer studies, the pooled study was conducted in North America and Europe, two in the USA, two in Europe and one in Asia; one study was restricted to pre-menopausal women, while the rest included both pre-menopausal and postmenopausal women. Of the eighteen publications, two were on ovarian cancer, of which one was a pooled study. Among the ovarian cancer studies, the pooled study was conducted in North America and Europe and one in Europe; all studies included a mixed population of pre-menopausal and postmenopausal women. Of the eighteen publications, ten were on prostate cancer, of which six were on total prostate cancer and four on fatal prostate cancer. Among the prostate cancer studies, six were conducted in the USA, two in Europe and two in Asia. While all studies on breast and ovarian cancers were adjusted for multiple potential confounders, three studies on prostate cancer were adjusted for age only.

\section{Breast cancer}

In the linear dose-response meta-analysis, five cohort studies $^{(36-40)}$ and one pooled study ${ }^{(26)}$ of five cohort studies were included, involving a total of 16023 cases with categoryspecific midpoints of egg consumption ranging from 0 to 8.8 eggs/week. The summary RR for an increase of 5 eggs consumed/week was 1.05 (95\% CI 0.99, 1.11), with no evidence of heterogeneity $\left(I^{2}=0 \%, \quad P_{\text {heterogeneity }}=0.93\right)$ (Fig. 2(a)). The results did not change materially when the serving size of egg intake was set to two eggs rather than one egg for studies ${ }^{(37-39)}$ that did not report or specify the serving size of egg intake on FFQ (data not shown). In sensitivity analyses excluding one study at a time, the results were robust to the influence of any single study included. Small-study 


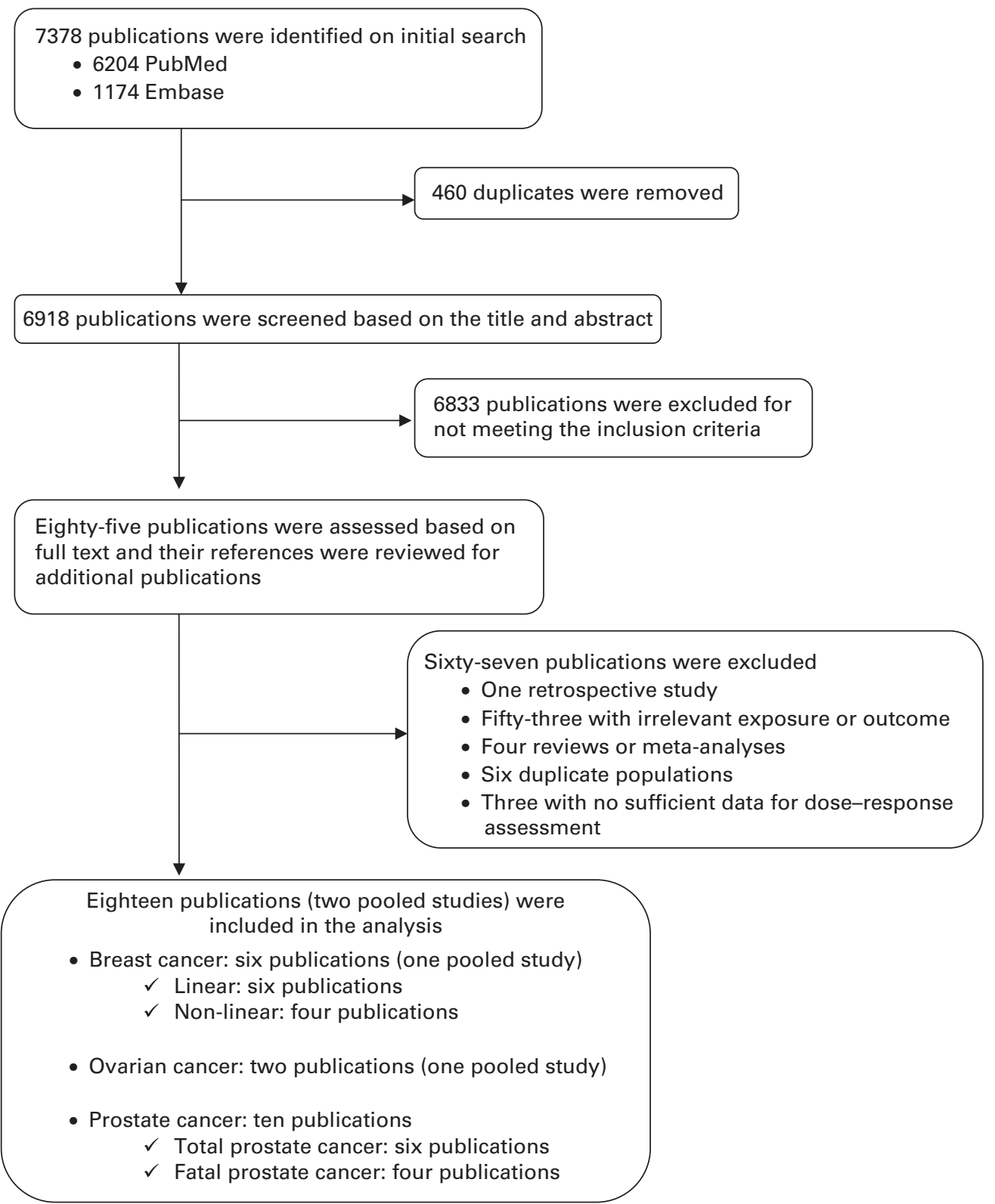

Fig. 1. Flow chart for study selection.

effects, such as publication bias, were not evident $\left(P_{\text {Egger }}=0.62\right)$.

The non-linear dose-response meta-analysis was conducted after excluding two studies ${ }^{(38,39)}$ that analysed egg intake in three categories only (15415 cases; range 0-8.8 eggs/week). While there was no evidence of non-linearity $\left(P_{\text {non-linearity }}=0.50 ;\right.$ Fig. 2(b)), intakes of $\geq 5$ eggs/week were significantly associated with an increased risk of breast cancer. Compared with no egg consumption, the summary RR was 1.04 (95\% CI 1.01, 1.07) for consuming 5 eggs/week and further increased to 1.09 (95\% CI 1.03, 1.15) for consuming about 9 eggs/week. In sensitivity analyses such as assuming one serving size of egg consumption as two eggs for the study by Gaard et $a l^{(37)}$ and excluding the study ${ }^{(37)}$, the results were found to be consistent, with the risk increasing significantly by $4 \%$ starting from intake of 5 eggs/week (data not shown)

\section{Ovarian cancer}

In the linear dose-response meta-analysis, a pooled study ${ }^{(41)}$ of eleven cohort studies and one independent cohort studies $^{(42)}$ (2636 cases; range of median egg intake of cohorts in the pooled analysis 0.8-3 eggs/week; category-specific midpoints of egg intake in one independent study $0.5-2.5$ eggs/week) were included. An increase of 5 eggs consumed/ week was associated with an approximately 9\% increased risk of ovarian cancer, but the association was not statistically significant (RR 1.09, 95\% CI $0.96, \quad 1.24 ; \quad I^{2}=3 \%$; $\left.P_{\text {heterogeneity }}=0.31\right)$ (Fig. 3). 
(a) First author, year

RR $\quad(95 \% \mathrm{Cl})$

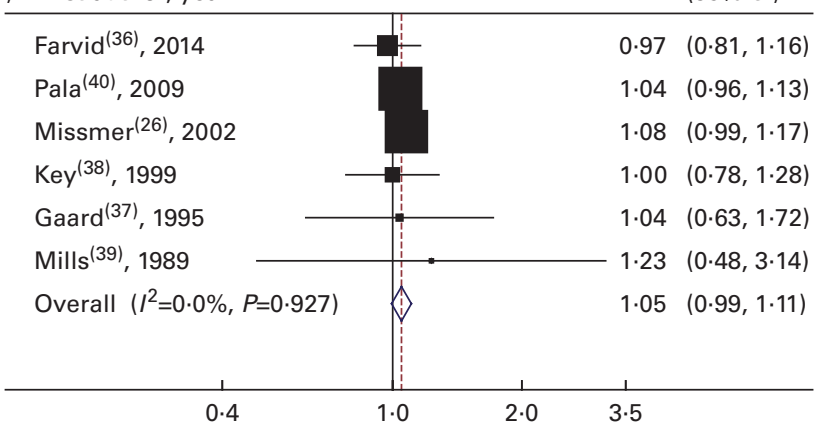

RR for an increase of 5 eggs consumed per week

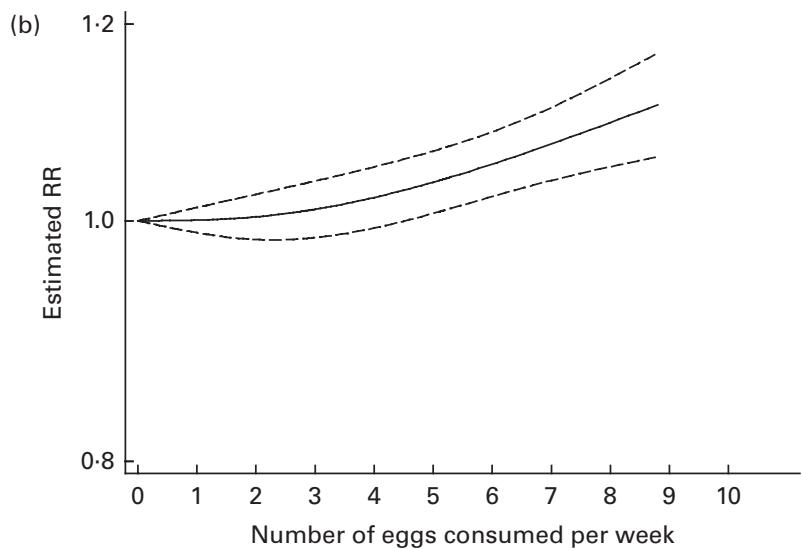

Fig. 2. Egg consumption and the risk of breast cancer: (a) linear doseresponse meta-analyses and (b) non-linear dose-response meta-analysis (reference: 0 eggs/week, $P_{\text {non-linearity }}=0.50$ ). RR, relative risk. (a) Although the Adventist Health Study was a participating cohort in the pooled study (Missmer, 2002), it was excluded from the egg analysis in the pooled study. Thus, the present meta-analysis included a study published from the Adventist Health Study (Mills, 1989). (b) The inner tick marks on the $x$-axis represent data points contributed by the studies included in the meta-analysis. Due to the overlap on the level of egg intake across some data points, the number of tick marks does not correspond to the number of data points. Best-fitting cubic spline; -.-., $95 \% \mathrm{Cl}$. A colour version of this figure can be found online at http://www.journals.cambridge.org/bjn

Due to a dominant influence the pooled study exerts on the summary estimate and limited number of studies included, small study effects, such as publication bias, and potential non-linear relationship were not tested.

\section{Prostate cancer}

A total of ten studies ${ }^{(25,43-51)}$ were eligible for the doseresponse meta-analysis, of which six studies $(43,44,47-49,51)$ investigated total prostate cancer (3655 cases; range 0.5-7 eggs/d) and the remaining four studies ${ }^{(25,45,46,50)}$ examined fatal prostate cancer (609 cases; range 0.3-6.8 eggs/d).

For total prostate cancer, there was no evidence of a linear association (RR 1.00, 95\% CI $0.88,1 \cdot 14, \quad I^{2}=0 \%$, $P_{\text {heterogeneity }}=0.69 ;$ Fig. 4$)$. In sensitivity analyses such as assigning two eggs to each frequency of egg consumption for studies ${ }^{(44,47,49)}$ that needed the serving size assumption and omitting one study at a time, the results did not change materially. Small-study effects, such as publication bias, were not evident $\left(P_{\text {Egger }}=0.72\right)$
In contrast, for fatal prostate cancer, an increase of 5 eggs consumed/week was associated with an approximately $47 \%$ elevated risk (RR 1.47, 95\% CI $1 \cdot 01,2 \cdot 14, I^{2}=40 \%$, $P_{\text {heterogeneity }}=0 \cdot 17$; Fig. 4$)$. When the serving size of egg consumptions was changed from one egg to two eggs for studies ${ }^{(45,46,50)}$ that needed such assumption, the effect size was attenuated (RR 1.23, 95\% CI 0.97, 1.55, $I^{2}=70 \%$, $P_{\text {heterogeneity }}=0.08$ ). In sensitivity analyses omitting one study at a time, a direct linear association persisted across each exclusion, but statistical significance was lost in all cases except for the exclusion of the study by Hsing et al. ${ }^{(45)}$. Evidence of small-study effects, such as publication bias, was evident $\left(P_{\text {Egger }}=0.04\right)$, with relatively smaller studies reporting a stronger linear association (Fig. 5).

The non-linear dose-response relationship was not explored due to the insufficient number of studies eligible for the robust estimation of the curve, which was specified a priori in the Methods section.

\section{Discussion}

Eggs have high contents of cholesterol and choline, and provide relatively high amounts of protein per energy content, all of which may link egg consumption to the risk of breast, ovarian and prostate cancers. However, most of the previous studies on such cancers have not investigated egg consumption as the primary exposure of interest, limiting a rigorous evaluation of the hypothesised associations. In the present linear dose-response meta-analysis of prospective observational studies, we found a statistically non-significant positive association with breast and ovarian cancers and a possible positive association with fatal prostate cancer, with an increase of 5 eggs consumed/week elevating the risk by 5,9 and $47 \%$, respectively. For total prostate cancer, no evidence of a linear association was found. In the case of breast cancer for which the non-linear dose-response meta-analysis was conducted, while evidence of non-linearity was not statistically significant, the curve showed an upward tendency, with women consuming $\geq 5$ eggs/week having a statistically significant but modestly elevated risk compared with non-consumers.

To date, one meta-analysis ${ }^{(18)}$ and one pooled study ${ }^{(26)}$ have been conducted to assess the relationship between egg intake and the risk of breast cancer. While findings from the

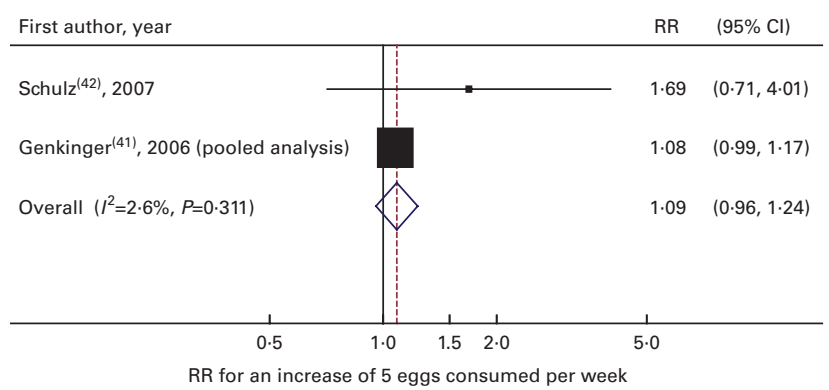

Fig. 3. Egg consumption and the risk of ovarian cancer. RR, relative risk. A colour version of this figure can be found online at http://www.journals. cambridge.org/bjn 


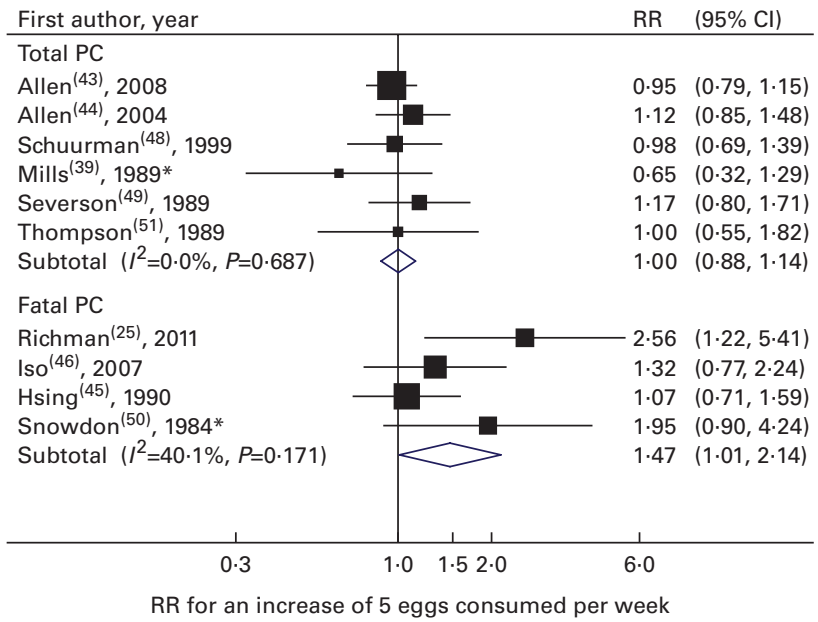

Fig. 4. Linear dose-response meta-analyses of egg consumption and the risk of prostate cancer (PC). ${ }^{*}$ Studies were from the same cohort (Adventist Health Study). RR, relative risk. A colour version of this figure can be found online at http://www.journals.cambridge.org/bjn

two different methods of analysis are conflicting, the present study may provide some clues to reconcile such inconsistency. The pooled analysis ${ }^{(26)}$ of five cohort studies suggested a J-shape association, with the risk decreasing for $<0$ to $<2$ eggs consumed/week (RR 0.93, 95\% CI 0.82, 1.05) but increasing for $>7$ eggs consumed/week (RR 1·07, $95 \%$ CI 0.90, 1·28) compared with no egg consumption. However, the metaanalysis $^{(18)}$ that included the pooled study, cohort studies and case-control studies did not confirm the J-shape association and reported an increased risk only in the intake categories of $2-5$ eggs/week relative to $<1$ egg/week. Although evidence for non-linearity was not statistically significant, the present study based on the pooled study and cohort studies found some upward tendency towards the dose-response curve, with the risk elevating statistically significantly for consuming $\geq 5$ eggs/week relative to 0 eggs/week, which is more consistent with the results obtained from the pooled analysis. Based on more than twice as many cases as the pooling project and with more data over the range of 3-6 eggs consumed/week contributed by the additional cohort studies, our analysis had richer information to examine the dose-response relationship. Inconsistency with the previous meta-analysis may be partially explained by its inclusion of case-control studies that are more prone to recall and selection biases and by its failure to account for correlation across the RR within the same study (e.g. two RR from the same study were pooled together with RR from other studies using a random-effects model).

For ovarian cancer, while no new study was identified to update the previous dose-response meta-analysis by the WRCF/AICR ${ }^{(52)}$, our analysis based on different inclusion criteria concerning the pooled analysis ${ }^{(41)}$ may contribute to raising the level of evidence for a positive association from the current 'limited - no conclusion' to 'limited - suggestive'. Of the eleven cohort studies included in the pooled analysis, four ${ }^{(53-56)}$ published the results separately. Unlike our analysis that included the pooled study ${ }^{(41)}$ itself, the analysis conducted by the WRCF/AICR included these four studies ${ }^{(53-56)}$. As eggs are a source of protein and fat, the relationship between egg intake and the risk of ovarian cancer has been investigated primarily in the context of examining the effect of either protein or fat intake on the risk of ovarian cancer. Thus, studies with a null finding would have been less likely to be published, which is a major source of publication bias in meta-analysis. Indeed, two ${ }^{(53,54)}$ of the four studies ${ }^{(53-56)}$ published independently from the pooled study ${ }^{(41)}$ reported a statistically significant, strong association. Additionally, as a pooled study combines data from the participating cohorts in a standardised manner, inclusion of pooled studies in meta-analyses helps reduce artificial heterogeneity arising from methodological differences across studies. The RR reported by the WRCF/AICR, after converting to the RR for an increase of 5 eggs consumed/ week, was 1.30 (95\% CI $\left.0.93,1.82, I^{2}=46 \%\right)$, which is stronger in the strength of association but less precise and more heterogeneous compared with our findings (RR 1.09, $95 \%$ CI $\left.0 \cdot 96,1 \cdot 24 ; I^{2}=3 \%\right)$.

Concerning prostate cancer, due to the lack of additional studies published, our findings are virtually identical to those reported by the WRCF/AICR ${ }^{(57)}$, suggesting that egg intake may have no effect on total prostate cancer but possibly increase the risk of fatal prostate cancer. While the evidence of small-study effects and the lack of comprehensive adjustment for potential confounders in the studies included warrant cautious interpretation, the observed positive association between egg intake and the risk of fatal prostate cancer is biologically plausible because cholesterol and, particularly, choline have been implicated in prostate cancer progression. In addition to serving as a precursor to androgens that promote prostate cancer growth, cholesterol is an essential component of animal cell membranes ${ }^{(5)}$. Thus, a large amount of cholesterol is required for malignant cells to support their rapid growth and proliferation ${ }^{(58)}$. In prostate cells, cholesterol accumulates as the cells transform to malignant tumours ${ }^{(59)}$. The use of a cholesterol-lowering drug, statins, was statistically significantly associated with an approximately $20 \%$ reduced risk of

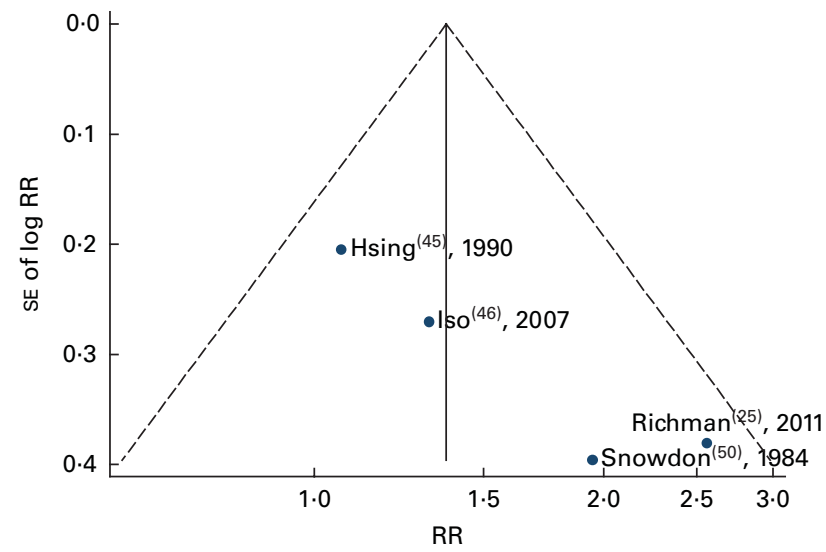

Fig. 5. Funnel plot with pseudo $95 \% \mathrm{Cl}$ for the linear dose-response meta-analysis of egg consumption and the risk of fatal prostate cancer. $\mathrm{RR}$, relative risk. A colour version of this figure can be found online at http:// www.journals.cambridge.org/bjn 
advanced prostate cancer ${ }^{(60)}$. Similarly, choline, through its conversion to a phospholipid by choline kinase, plays an important role in cell membrane synthesis and function, thereby implicated in cancer proliferation and progression ${ }^{(11)}$. Malignant prostate cells overexpress choline transporter and kinase for increased choline uptake and metabolism ${ }^{(11,61)}$, and choline is more highly concentrated in malignant than in normal prostate cells ${ }^{(62)}$. Considering that choline is an essential nutrient that must be consumed through diet and that eggs are a major contributor to choline intake ${ }^{(63)}$, contribution of choline appears to be particularly important in linking egg intake and the risk of fatal prostate cancer.

The present meta-analysis has several limitations. First, measurement error in egg intake is of particular concern. Given the growing consumption of eggs globally ${ }^{(1)}$, participants may have changed their egg consumption over time. However, most of the included studies with a long-term follow-up based their analysis on baseline egg intake only. Additional measurement errors were introduced during the dose-response meta-analysis due to inevitable assumptions such as assigning one egg to each serving of egg intake for studies that did not report or specify the serving size of egg intake on FFQ; using the midpoint of egg intake in each category as the dose for corresponding RR; and approximating the width of the open-ended highest category from the adjacent interval. Such inevitable measurement errors from diverse sources could bias the results in either direction, but are generally anticipated to attenuate the true effect ${ }^{(64)}$, particularly since the dietary information was collected before the participants' knowledge of case status. The concern is further alleviated, given the consistency in the present results regardless of the number of eggs (one or two) assigned assigned to each serving of egg intake for some studies that needed the serving size assumption.

Second, while the proportion of eggs consumed as a component of recipes rather than in-shell is increasing, especially in developed countries ${ }^{(1)}$, most of the included studies analysed only egg consumed as such, underestimating the true egg intake. Thus, our linear dose-response meta-analysis may have overestimated the true association and, particularly for breast cancer for which a non-linear relationship was explored, the cut-off point from which a statistically significantly elevated risk starts may occur at a total intake $\geq 5$ eggs/week. However, as egg consumed as such is likely to be the major determinant of the variation in total egg intake, quantifying cancer risk based on egg consumption as such still provides useful information.

Third, most of the studies included did not adjust for dietary factors. While not many dietary factors are established risk factors for the cancers examined, people eat a food item not in isolation but in combination with other foods. Thus, as a potential explanation for the statistically significant associations observed between egg intake and the risk of breast and fatal prostate cancers, confounding by foods (e.g. red meat) correlated with egg intake cannot be ruled out completely.

Finally, while there was no evidence of statistical heterogeneity in summary RR for breast, ovarian and total prostate can- cers, we were not able to explore some important biological heterogeneity due to a lack of data. For instance, it has been reported that the association of egg intake and the risk of breast cancer is stronger among pre-menopausal women than among postmenopausal women ${ }^{(65)}$, and that egg intake is specifically associated with the risk of breast cancer with positive oestrogen and progesterone receptors ${ }^{(66)}$. Furthermore, in light of the evidence that an increment in serum cholesterol for a given dietary cholesterol intake diminishes with increasing baseline dietary cholesterol intake ${ }^{(67)}$ and that diabetic people are more responsive to dietary cholesterol intake $^{(68)}$, if cholesterol is the main mediator of the relationship between egg intake and the risk of cancers, the association might be stronger in populations with otherwise low dietary cholesterol intake or in diabetic patients.

However, there are also several strengths of the present meta-analysis. To date, this is the first meta-analysis that attempted to summarise the dose-response relationships of egg intake with breast, prostate and gynaecological cancers. As we excluded case-control studies, our findings are more robust against recall bias and selection bias. Low heterogeneity observed in most of the analyses increases the generalisability of our findings. By preferentially incorporating pooled studies where possible, our estimates had more precision, less unwanted heterogeneity, and more immunity against publication bias than those that would have been obtained by incorporating selectively published studies from a few cohorts included in a pooled study. Finally, our dose-response metaanalysis for total prostate cancer included studies that were conducted in Europe and Asia, where the prostate-specific antigen screening rate is relatively low, and in the USA, before 1994, when only a minority of men were screened for prostate-specific antigen. Hence, total prostate cancer is likely to represent more of clinically significant prostate cancer rather than indolent prostate cancer.

In conclusion, consuming $\geq 5$ eggs/week may be associated with an elevated risk of breast cancer compared with no egg consumption. The present study provides only limited evidence to support a direct linear association between egg intake and the risk of ovarian and fatal prostate cancers. While our conclusion is tempered by the potential for publication bias and confounding by other foods, we cannot rule out an association. Considering that eggs are frequently consumed worldwide, more prospective studies primarily investigating the effect of egg consumption on the risk of these cancers are warranted to confirm these associations.

\section{Supplementary material}

To view supplementary material for this article, please visit http://dx.doi.org/10.1017/S0007114515002135

\section{Acknowledgements}

The authors' contributions are as follows: N. K. designed the study, analysed and interpreted the data, and drafted the manuscript; D. H. L., N. M., H. O. and H. L. acquired, analysed and interpreted the data; D. A. interpreted the data and drafted 
the manuscript; D. C. G. wrote the statistical analysis code, interpreted the data and drafted the manuscript; E. L. G. was the principal investigator who designed the study, interpreted the results and drafted the manuscript.

The authors declare that they have no conflicts of interest.

\section{References}

1. Food and Agriculture Organization of the United Nations (2010) Agribusiness Handbook: Poultry Meat and Eggs. Available at: https://www.responsibleagroinvestment.org/ node/738 (accessed 1 September 2014).

2. U.S. Department of Agriculture, Agricultural Research Service (2010) USDA National Nutrient Database for Standard Reference, Release 23, Nutrient Data Laboratory Home Page. http://www.ars.usda.gov/nutrientdata (accessed September 2014).

3. US Food and Drug Administration (2013) Guidance for industry: a food labeling guide (14. Appendix F: calculate the percent daily value for the appropriate nutrients). http://www.fda.gov/downloads/Food/GuidanceRegulation/ UCM265446.pdf (accessed September 2014).

4. Food and Nutrition Board of Institute of Medicine, National Academies. Dietary Reference Intakes for Folate and Other B Vitamins. http://ods.od.nih.gov/Health_Information/ Dietary_Reference_Intakes.aspx (accessed September 2014).

5. Berg JM, Tymoczko JL \& Stryer L, (2002) Biochemistry. In Section 26.4. Important Derivatives of Cholesterol Include Bile Salts and Steroid Hormones, 5th ed New York: W.H. Freeman; available at: http://www.ncbi.nlm.nih.gov/books/ NBK22339/.

6. Henderson BE \& Feigelson HS (2000) Hormonal carcinogenesis. Carcinogenesis 21, 427-433.

7. Bosland MC \& Mahmoud AM (2011) Hormones and prostate carcinogenesis: androgens and estrogens. J Carcinog 10, 33.

8. Yager JD \& Davidson NE (2006) Estrogen carcinogenesis in breast cancer. $N$ Engl J Med 354, 270-282.

9. Nelson ER, Wardell SE, Jasper JS, et al. (2013) 27-Hydroxycholesterol links hypercholesterolemia and breast cancer pathophysiology. Science 342, 1094-1098.

10. DuSell CD, Umetani M, Shaul PW, et al. (2008) 27-Hydroxycholesterol is an endogenous selective estrogen receptor modulator. Mol Endocrinol 22, 65-77.

11. Awwad HM, Geisel J \& Obeid R (2012) The role of choline in prostate cancer. Clin Biochem 45, 1548-1553.

12. Zhang CX, Pan MX, Li B, et al. (2013) Choline and betaine intake is inversely associated with breast cancer risk: a two-stage case-control study in China. Cancer Sci 104, $250-258$

13. Giovannucci E, Pollak M, Liu Y, et al. (2003) Nutritional predictors of insulin-like growth factor I and their relationships to cancer in men. Cancer Epidemiol Biomarkers Prev 12, 84-89.

14. Maskarinec G, Takata Y, Murphy SP, et al. (2005) Insulin-like growth factor-1 and binding protein-3 in a 2-year soya intervention among premenopausal women. Br J Nutr 94, $362-367$.

15. Giovannucci E (1999) Insulin-like growth factor-I and binding protein-3 and risk of cancer. Horm Res 51, Suppl. 3, $34-41$.

16. Underwood LE, Thissen JP, Lemozy S, et al. (1994) Hormonal and nutritional regulation of IGF-I and its binding proteins. Horm Res 42, 145-151.

17. Salehi F, Turner MC, Phillips KP, et al. (2008) Review of the etiology of breast cancer with special attention to organo- chlorines as potential endocrine disruptors. I Toxicol Environ Health B Crit Rev 11, 276-300

18. Si R, Qu K, Jiang Z, et al. (2014) Egg consumption and breast cancer risk: a meta-analysis. Breast Cancer 21, 251-261.

19. Zeng ST, Guo L, Liu SK, et al. (2015) Egg consumption is associated with increased risk of ovarian cancer: evidence from a meta-analysis of observational studies. Clin Nutr 34, 635-641.

20. Bandera EV, Kushi LH, Moore DF, et al. (2007) Consumption of animal foods and endometrial cancer risk: a systematic literature review and meta-analysis. Cancer Causes Control 18, 967-988.

21. Xie B \& He HD (2012) No association between egg intake and prostate cancer risk: a meta-analysis. Asian Pac J Cancer Prev 13, 4677-4681.

22. World Cancer Research Fund/American Institute for Cancer Research (2014) Continuous Update Project Report. Food, Nutrition, Physical Activity, and the Prevention of Ovarian Cancer 2014. http://www.dietandcancerreport.org/ cancer_resource_center/downloads/cu/Ovarian-Cancer-2014Report.pdf (accessed September 2014).

23. World Cancer Research Fund International/American Institute for Cancer Research (2014) Continuous Update Project Report: Diet, Nutrition, Physical Activity, and Prostate Cancer. www.wcrf.org/sites/default/files/ProstateCancer-2014-Report.pdf (accessed January 2015).

24. Stroup DF, Berlin JA, Morton SC, et al. (2000) Meta-analysis of observational studies in epidemiology: a proposal for reporting. Meta-analysis Of Observational Studies in Epidemiology (MOOSE) group. JAMA 283, 2008-2012.

25. Richman EL, Kenfield SA, Stampfer MJ, et al. (2011) Egg, red meat, and poultry intake and risk of lethal prostate cancer in the prostate-specific antigen-era: incidence and survival. Cancer Prev Res (Phila) 4, 2110-2121.

26. Missmer SA, Smith-Warner SA, Spiegelman D, et al. (2002) Meat and dairy food consumption and breast cancer: a pooled analysis of cohort studies. Int J Epidemiol 31, 78-85.

27. Greenland S \& Longnecker MP (1992) Methods for trend estimation from summarized dose-response data, with applications to meta-analysis. Am J Epidemiol 135, 1301-1309.

28. Harrell FE Jr, Lee KL \& Pollock BG (1988) Regression models in clinical studies: determining relationships between predictors and response. J Natl Cancer Inst 80, 1198-1202.

29. Orsini N \& Greenland S (2011) A procedure to tabulate and plot results after flexible modeling of a quantitative covariate. Stata J 11, 1-29.

30. White IR (2009) Multivariate random-effects meta-analysis. Stata J 9, 40-56.

31. Higgins JP \& Thompson SG (2002) Quantifying heterogeneity in a meta-analysis. Stat Med 21, 1539-1558.

32. Higgins JP, Thompson SG, Deeks JJ, et al. (2003) Measuring inconsistency in meta-analyses. BMJ 327, 557-560.

33. Sterne JA, Gavaghan D \& Egger M (2000) Publication and related bias in meta-analysis: power of statistical tests and prevalence in the literature. J Clin Epidemiol 53, 1119-1129.

34. Nuesch E, Trelle S, Reichenbach S, et al. (2010) Small study effects in meta-analyses of osteoarthritis trials: meta-epidemiological study. BMJ 341, c3515.

35. Harbord RM, Egger M \& Sterne JAC (2006) A modified test for small-study effects in meta-analyses of controlled trials with binary endpoints. Stat Med 25, 3443-3457.

36. Farvid MS, Cho E, Chen WY, et al. (2014) Dietary protein sources in early adulthood and breast cancer incidence: prospective cohort study. BMJ 348, g3437. 
37. Gaard M, Tretli S \& Loken EB (1995) Dietary fat and the risk of breast cancer: a prospective study of 25,892 Norwegian women. Int J Cancer 63, 13-17.

38. Key TJ, Sharp GB, Appleby PN, et al. (1999) Soya foods and breast cancer risk: a prospective study in Hiroshima and Nagasaki. Japan. Br J Cancer 81, 1248-1256.

39. Mills PK, Beeson WL, Phillips RL, et al. (1989) Dietary habits and breast cancer incidence among Seventh-day Adventists. Cancer 64, 582-590.

40. Pala V, Krogh V, Berrino F, et al. (2009) Meat, eggs, dairy products, and risk of breast cancer in the European Prospective Investigation into Cancer and Nutrition (EPIC) cohort. Am J Clin Nutr 90, 602-612.

41. Genkinger JM, Hunter DJ, Spiegelman D, et al. (2006) A pooled analysis of 12 cohort studies of dietary fat, cholesterol and egg intake and ovarian cancer. Cancer Causes Control 17, 273-285.

42. Schulz M, Nothlings U, Allen N, et al. (2007) No association of consumption of animal foods with risk of ovarian cancer. Cancer Epidemiol Biomarkers Prev 16, 852-855.

43. Allen NE, Key TJ, Appleby PN, et al. (2008) Animal foods, protein, calcium and prostate cancer risk: the European Prospective Investigation into Cancer and Nutrition. Br J Cancer 98, 1574-1581.

44. Allen NE, Sauvaget C, Roddam AW, et al. (2004) A prospective study of diet and prostate cancer in Japanese men. Cancer Causes Control 15, 911-920.

45. Hsing AW, McLaughlin JK, Schuman LM, et al. (1990) Diet, tobacco use, and fatal prostate cancer: results from the Lutheran Brotherhood Cohort Study. Cancer Res 50 , 6836-6840.

46. Iso H, Kubota $\mathrm{Y}$ \& Japan Collaborative Cohort Study for Evaluation of Cancer (2007) Nutrition and disease in the Japan Collaborative Cohort Study for Evaluation of Cancer (JACC). Asian Pac J Cancer Prev Suppl. 8, 35-80.

47. Mills PK, Beeson WL, Phillips RL, et al. (1989) Cohort study of diet, lifestyle, and prostate cancer in Adventist men. Cancer 64, 598-604.

48. Schuurman AG, van den Brandt PA, Dorant E, et al. (1999) Animal products, calcium and protein and prostate cancer risk in The Netherlands Cohort Study. Br J Cancer 80, $1107-1113$

49. Severson RK, Nomura AM, Grove JS, et al. (1989) A prospective study of demographics, diet, and prostate cancer among men of Japanese ancestry in Hawaii. Cancer Res 49, $1857-1860$

50. Snowdon DA, Phillips RL \& Choi W (1984) Diet, obesity, and risk of fatal prostate cancer. Am J Epidemiol 120, 244-250.

51. Thompson MM, Garland C, Barrettconnor E, et al. (1989) Heart disease risk factors, diabetes, and prostatic cancer in an adult community. Am J Epidemiol 129, 511-517.

52. World Cancer Research Fund/American Institute for Cancer Research (2013) WCRF/AICR Systematic Literature Review Continuous Update Project Report. The Associations between Food, Nutrition, Physical Activity, and the Risk of Ovarian Cancer 2013. http://www.dietandcancerreport.org/ cancer_resource center/downloads/cu/Ovarian-Cancer-SLR2013.pdf (accessed January 2015).

53. Bertone ER, Rosner BA, Hunter DJ, et al. (2002) Dietary fat intake and ovarian cancer in a cohort of US women. $A m J$ Epidemiol 156, 22-31.

54. Kushi LH, Mink PJ, Folsom AR, et al. (1999) Prospective study of diet and ovarian cancer. Am J Epidemiol 149, 21-31.

55. Kiani F, Knutsen S, Singh P, et al. (2006) Dietary risk factors for ovarian cancer: the Adventist Health Study (United States). Cancer Causes Control 17, 137-146.

56. Larsson SC \& Wolk A (2005) No association of meat, fish, and egg consumption with ovarian cancer risk. Cancer Epidemiol Biomarkers Prev 14, 1024-1025.

57. World Cancer Research Fund International/American Institute for Cancer Research (2014) World Cancer Research Fund International Systematic Literature Review: The Associations between Food, Nutrition, Physical Activity, and the Risks of Prostate Cancer. http://www.wcrf.org/sites/default/ files/Prostate-Cancer-SLR-2014.pdf (accessed January 2015).

58. Pelton K, Freeman MR \& Solomon KR (2012) Cholesterol and prostate cancer. Curr Opin Pharmacol 12, 751-759.

59. Freeman MR \& Solomon KR (2004) Cholesterol and prostate cancer. J Cell Biochem 91, 54-69.

60. Bansal D, Undela K, D'Cruz S, et al. (2012) Statin use and risk of prostate cancer: a meta-analysis of observational studies PLOS ONE 7, e46691.

61. Muller SA, Holzapfel K, Seidl C, et al. (2009) Characterization of choline uptake in prostate cancer cells following bicalutamide and docetaxel treatment. Eur J Nucl Med Mol Imaging 36, 1434-1442.

62. Ackerstaff E, Pflug BR, Nelson JB, et al. (2001) Detection of increased choline compounds with proton nuclear magnetic resonance spectroscopy subsequent to malignant transformation of human prostatic epithelial cells. Cancer Res $\mathbf{6 1}$, 3599-3603.

63. Chester DN, Goldman JD \& Ahuja JK, et al. (2011) Dietary Intakes of Choline: What We Eat in America, NHANES 2007-2008. Food Surveys Research Group Dietary Data Brief No. 9. October 2011. http://www.ars.usda.gov/ SP2UserFiles/Place/80400530/pdf/DBrief/9_choline_intakes_ 0708.pdf (accessed January 2015).

64. Freedman LS, Schatzkin A, Midthune D, et al. (2011) Dealing with dietary measurement error in nutritional cohort studies. J Natl Cancer Inst 103, 1086-1092.

65. Holmes MD, Colditz GA, Hunter DJ, et al. (2003) Meat, fish and egg intake and risk of breast cancer. Int J Cancer 104, $221-227$.

66. Wirfalt E, Li C, Manjer J, et al. (2011) Food sources of fat and sex hormone receptor status of invasive breast tumors in women of the Malmo Diet and Cancer cohort. Nutr Cancer 63, 722-733.

67. Hopkins PN (1992) Effects of dietary cholesterol on serum cholesterol: a meta-analysis and review. Am J Clin Nutr $\mathbf{5 5}$, 1060-1070.

68. Franz MJ, Bantle JP, Beebe CA, et al. (2004) Nutrition principles and recommendations in diabetes. Diabetes care $\mathbf{2 7}$, Suppl. 1, S36-S46. 